\title{
EUROBONDS E CRISI DELL'EUROZONA (A proposito di un recente volume)
}

\author{
Nota del m.e. SILVIO BERETTA (*)
}

(Adunanza del 17 gennaio 2013)

SuNTO. - La nota collega l'emissione di eurobonds agli aspetti politici dell'attuale crisi dell'eurozona. Si sottolinea, in primo luogo, come sia l'eventuale futura emissione di eurobonds sia il rifiuto della stessa testimonino dell'instaurarsi di rapporti cooperativi, o non cooperativi, all'interno dell'area euro. Tale alternativa, a sua volta, testimonia della natura essenzialmente politica della crisi in atto. In questa prospettiva, l'esigenza di un'integrazione politica completa in quanto fattore decisivo di uscita dalla crisi viene analizzata esaminando alcune prese di posizione, in particolare le conclusioni del Gruppo di riflessione sul futuro dell'UE e le considerazioni finali del Governatore della Banca d'Italia.

$* * *$

ABSTRACT. - The paper connects the eurobonds issue to the political aspects of the current eurozone crisis. It firstly emphasizes that the possible future issuance of Eurobonds, or the refusal to do so, represent signs of the emergence of cooperative or, alternatively, non-cooperative behaviors among eurozone countries. Such a dichotomy, in turn, appears as a clear proof of the political roots of the crisis. Accordingly to this perspective, the persistent demand for a full European political integration as the decisive step out of the crisis is reviewed by analyzing a list of works, particularly the conclusions of the Reflection Group on the future of the European Union and the concluding remarks of the Governor of the Bank of Italy.

1. La nota che segue trae spunto dalla recente pubblicazione di un volume ${ }^{1}$ che raccoglie, rielaborati, saggi discussi in forma di relazione

(*) Università degli Studi di Pavia, Italy.

E-mail: silvio.beretta@unipv.it

1 S. Beretta, F. Osculati (a cura), Verso un debito pubblico europeo?, Soveria Mannelli, Rubbettino, 2012. 
in un seminario svoltosi il 15 aprile 2011 presso la Facoltà di Scienze politiche dell'Università di Pavia, con l'aggiunta tuttavia di contributi ulteriori intesi tanto a "contestualizzare" quel dibattito quanto ad approfondirne i profili analitici: argomento erano gli eurobond, tema da tempo oggetto di discussione, nonché "segno di contraddizione" fra concezioni dell'Unione Europea - e quindi fra "traiettorie" politico-istituzionali dell'Unione stessa - fra loro divergenti.

La sede di svolgimento dell'incontro - Pavia appunto - era a sua volta evocativa, essendo stata da decenni, e continuando a essere, luogo di elaborazione e di promozione dell'idea e delle istituzioni dell'Europa federale alla ricerca di soluzioni, sia politico-istituzionali sia tecniche, che la crisi in atto rendeva, come rende tuttora, nel contempo più urgenti da perseguire e più ardue da conseguire: caratteristica questa la difficile necessità - che ha contraddistinto tante tappe della costruzione dell'Europa unita. L'Ateneo pavese e Pavia sono state infatti il luogo di lavoro di Mario Albertini (nonché del prestigioso cenacolo di federalisti che con lui militavano) al quale è legata molta parte della riflessione contemporanea sui temi del federalismo ma anche dell'azione politica per l' Europa federale. A Pavia era stata allora da poco completata, a cura di Nicoletta Mosconi, l'imponente edizione - nove volumi - degli scritti di Albertini, ${ }^{2}$ cronaca straordinaria della militanza cinquantennale di uno studioso in favore, come sottolinea la curatrice, di una "...nuova cultura politica di cui il mondo ha bisogno, la cultura politica dell'unità del genere umano, per avviarsi verso il superamento dell'ineguale distribuzione del potere e della ricchezza fra i popoli e verso il governo razionale del mondo". ${ }^{3}$ Vale la pena di richiamare qui i quattro punti enunciati da Albertini in una dichiarazione del 1995. Il primo: "Per quanto riguarda l'Europa non serve la divisione italiana in parti ma l'unità nazionale". Il secondo: "In questione è il fatto che con la ratifica del Trattato di Maastricht i cittadini italiani sono ormai anche cittadini europei”. Il terzo:

“...col referendum europeo del 1989...l'88\% degli italiani si è espresso a favore della Costituzione europea. Ne segue che ogni

2 M. Albertini, Tutti gli scritti, Voll. I - IX, Bologna, il Mulino, 2006-2010.

3 N. Mosconi, Premessa in M. Albertini, Tutti gli scritti, Vol. VII, 1976-1978, Bologna, il Mulino, 2009, p. 17. 
scelta politica che divide i partiti sul terreno europeo è antidemocratica e illegittima". Il quarto: "Va detto...che coloro che non hanno perso il senso della realtà sanno che la degenerazione della lotta per il potere in Italia è ormai giunta ad un punto tale che può essere sanata solo con la piena assunzione da parte dell'Italia del ruolo europeo che ebbe con De Gasperi e con Spinelli". ${ }^{4}$

Nel presentare poi l'opera di Francesco Rossolillo, un altro esponente di spicco del federalismo italiano ed europeo che ha operato a Pavia, Giovanni Vigo ricorda che: “...si è creata nel corso degli anni una situazione che mette in pericolo lo sbocco federale e lo rende sempre più urgente... Si assiste cioè al progressivo indebolimento della compattezza e della capacità decisionale dell'Unione in seguito ai successivi allargamenti, al riemergere della difesa degli interessi nazionali a scapito dell'interesse europeo e all'uscita di scena degli uomini politici che, dopo la seconda guerra mondiale, erano convinti che l'unificazione politica dell'Europa fosse la sola via di salvezza".

Un perché di fondo di tali difficoltà si ritrova poi in un' arguta frase pronunciata nel 2007 dal presidente dell'Eurogruppo, il lussemburghese Jean-Claude Juncker, citata in "The Economist" del 15 marzo di quell'anno: "Sappiamo tutti cosa fare, solo che non sappiamo come fare per essere rieletti una volta che lo abbiamo fatto". E vale la pena di richiamare qui un altro passo, dovuto anch'esso a un grande europeista, Tommaso Padoa-Schioppa. Da ministro del Governo italiano, e con riferimento ai lavori dell'Eurogruppo, Padoa-Schioppa affermava, sempre nel 2007: "L'Eurogruppo sta prestando una sempre maggiore attenzione alle riforme strutturali...Tuttavia l'approccio...si limita a comparare le esperienze fatte da diversi paesi, incoraggiando i ritardatari a imparare dai riformatori più attivi...Ciò che manca, io credo, è l'Unione. Se le riforme non vengono attuate, spesso la ragione risiede nel fatto che i governi si mettono d'accordo in questo senso. Lo abbia-

4 M. Albertini, L'Italia non si salva senza l'Europa, in Id., Tutti gli scritti, Vol. IX, 1985-1995, Bologna, il Mulino, 2010, p. 943. Si vuole ricordare qui che l'Università di Pavia ha conferito ad Altiero Spinelli la laurea h.c. in Scienze politiche.

5 G. Vigo, Introduzione in F. Rossolillo, Senso della storia e azione politica, 2 volumi, Bologna, il Mulino, 2009, p. 28. 
mo visto con l'energia, lo abbiamo visto con la finanza, lo abbiamo visto con i servizi...So quanto difficile e raro sia abbandonare un approccio comparativo per lavorare come una vera Unione. Penso, tuttavia, che sia di vitale importanza per le nostre economie comprendere che il destino della maggior parte delle riforme strutturali risieda a Bruxelles, non nelle capitali nazionali" ${ }^{6}$

2. Proporre le relazioni discusse in quel seminario a oltre un anno di distanza dal suo svolgimento ha tuttavia rappresentato una discreta sfida. Gli avvenimenti successivi a quella data (l'aprile 2011 appunto) rendevano infatti l'argomento, come le proposte formulate in quella sede, di particolare interesse e attualità. Pretendevano tuttavia, proprio in ragione di tale attualità, un inquadramento più documentato, attento anche ad altre prese di posizione, sia precedenti che contemporanee o immediatamente successive. Di alcune darò brevemente conto prima di esporre alcuni contenuti del volume.

Nel rassegnare - era il maggio 2010 - al Consiglio Europeo la propria relazione conclusiva, ${ }^{7}$ il Gruppo di riflessione sul futuro dell'UE insediato a fine 2007 (ne faceva parte Mario Monti, socio di questo Istituto Lombardo) identificava i principali snodi problematici della costruzione europea, formulando proiezioni al 2030; avanzava inoltre proposte circa le modalità di intervento più idonee. Ad avviso del Gruppo quegli snodi - significativamente tutti reali - riguardavano 1) la promozione del capitale umano, 2) la realizzazione di una politica energetica comune, 3) la lotta contro i cambiamenti climatici, 4) la necessità di fronteggiare la sfida demografica, 5) il perseguimento della sicurezza interna ed esterna sulla base di una visione "europea" della difesa collettiva. Rispetto all'urgenza di ciascuno di tali problemi, tuttavia, lo stato dell'Europa non appariva confortante agli estensori della relazione, tanto che, in apertura di questa, il Gruppo anticipava che "Le nostre conclusioni non sono rassicuranti né per l'Unione né per i nostri cittadini". ${ }^{8}$ Rispetto a ciascuna delle urgenze

6 Si tratta di un passo tratto da un'intervista rilasciata a Bruxelles il 15 marzo 2007.

7 Progetto Europa 2030. Sfide e opportunità, Relazione al Consiglio europeo del gruppo di riflessione sul futuro dell'UE 2030, Unione Europea, 2010.

8 Ivi, p. 3. 
identificate l'Unione appare infatti, ai componenti del gruppo di riflessione, non adeguatamente "attrezzata".

Se infatti quelle richiamate erano le sfide reali che l'Europa si trovava allora, e si trova tuttora, a dover fronteggiare, è al loro raggiungimento che dovevano, e dovrebbero, essere finalizzati i processi decisionali comunitari. Ma è proprio a questo proposito che il giudizio del Gruppo si fa severo. La crisi infatti “...iniziata sull'altra sponda dell'Atlantico, ha colpito l'Europa più di qualsiasi altra regione del mondo portando alla luce le debolezze strutturali dell'economia europea, da lungo tempo individuate ma troppo spesso ignorate". ${ }^{9} \mathrm{Ne}$ consegue che "Di fronte a una crisi che non hanno provocato, i cittadini continueranno a credere al progetto europeo solo se i leader saranno onesti sull'entità delle sfide che si prospettano e se essi saranno chiamati a uno sforzo paragonabile a quello che ha dato prosperità all'Europa dopo la seconda guerra mondiale" ${ }^{10}$ Completamento del mercato unico accompagnato da un migliore coordinamento fiscale e dal rafforzamento delle procedure di controllo sui bilanci nazionali, riforme del mercato del lavoro, modernizzazione delle pratiche del governo societario, riforma del funzionamento e della vigilanza degli istituti finanziari sono ambiti di intervento essenziali per "...rafforzare il buon governo economico nell'UE", ${ }^{11}$ in un contesto in cui euro e patto di stabilità e crescita non si sono rivelati adeguati ad assicurare un sufficiente grado di convergenza fra le economie. In questo quadro l'opinione del Gruppo anticipa chiaramente eventi e dibattiti successivi, che per altro faranno seguito a posizioni critiche già autorevolmente sostenute in passato. ${ }^{12}$
9 Ivi, p. 4.
10 Ivi, p. 7
11 Ivi, p. 4.

12 Da tempo infatti, e prima del $1^{\circ}$ gennaio 1999 quando l'euro entrò in vigore in undici dei quindici Stati allora membri dell'Unione, studiosi avevano mosso critiche ai parametri (3\% del rapporto disavanzo pubblico/Pil e 60\% del rapporto debito pubblico/Pil separatamente considerati) che un Protocollo allegato al Trattato di Maastricht individuava quali "valori di riferimento" per valutare la "sostenibilità" dei conti pubblici di un paese membro. Cfr. per tutti L.L. Pasinetti, The myth (or folly) of the 3\% deficit/GDP Maastricht "parameter" in "Cambridge Journal of Economics", 1998, 22, pp. 103-16. Vi si sottolinea fra l'altro: 1) che il valore di riferimento del parametro debito pubblico/Pil rappresenta semplicemente la media osservata dei valori rilevati nei paesi europei al momento della redazione del Trattato; 2 ) che il valore di riferimento del parametro disavanzo pubblico/Pil ha carattere di mero simbolo, ma nessun'altra 
"La prima priorità - si afferma - deve restare la creazione di posti di lavoro e la crescita", ${ }^{13}$ dal momento che "Se si taglia troppo presto la spesa, la ripresa potrebbe scivolare nella direzione opposta", ${ }^{14}$ e di conseguenza "...gli Stati membri che non possono più permettersi di spendere, per le costose operazioni di salvataggio, le spese sociali che aumentano e le fonti di gettito che si riducono, dovranno fare affidamento sull'iniziativa dell'UE e degli altri Stati membri per creare le condizioni per la ripresa economica". ${ }^{15}$

3. A un anno dal rapporto del Gruppo di riflessione, e a poche settimane dall'incontro pavese, il Governatore della Banca d'Italia

giustificazione; 3) che è invece individuabile per ogni paese un' area di sostenibilità dei conti pubblici compatibile con un infinito numero di valori dei due parametri, oltre che del tasso di sviluppo del Pil nominale, che il Trattato implicitamente ipotizzava pari al $5 \%$ annuo; 4) che, ai fini della sostenibilità dei conti pubblici, è cruciale la differenza fra tasso d'interesse e tasso di sviluppo del Pil; 5) che, accanto a quello del debito pubblico, rileva il livello del debito del settore privato dell'economia.

13 Progetto Europa 2030. Sfide e opportunità, Relazione al Consiglio europeo del gruppo di riflessione sul futuro dell'UE 2030, cit., p. 4. Riferendosi criticamente alle prescrizioni del Trattato a proposito del valore di riferimento del rapporto disavanzo pubblico/Pil, Pasinetti aveva già segnalato che "The whole of the Maastricht Treaty seems to have been reduced to the fulfilment of this symbolic figure of a 3\% public deficit/GDP ratio... But even symbols cannot escape the reality of their implications. If a $3 \%$ public deficit/GDP ratio is to be rigidly adhered to and regarded as a symbol of European fiscal and financial stability (even at the cost of heavy sacrifice [corsivo non nel testo, n.d.r.]), it surely should be an absolutely necessary condition for fiscal and financial stability. Nobody has never proved this. In fact it cannot be proved..." (L.L. Pasinetti, The myth (or folly) of the 3\% deficit/GDP Maastricht "parameter", cit., p. 104). In un successivo lavoro (L.L. Pasinetti, Public Debt in the European Union Countries: Two Ways of Facing the Problem in J. Krishnakumar, E. Ronchetti (a cura), Panel Data Econometrics: Future Directions. Papers in Honour of Professor Pietro Balestra, Amsterdam, Elseviers, 2000, pp. 317-28) Pasinetti, riferendosi al secondo "valore di riferimento" - il rapporto debito pubblico/Pil - e alle relative prescrizioni del Trattato, segnalerà (p. 327) - in contrasto con l'orientamento prevalente a favore della mera riduzione del debito stesso - la via alternativa per la quale "A reduction of the social burden of the public debt can be obtained... through internationally co-ordinated efforts... designed to drive interest rates down and growth rates up, thereby producing a stunning triple benefit: to reduce fiscal pressure, to boost GDP growth and to curb unemployment".

14 Progetto Europa 2030. Sfide e opportunità, Relazione al Consiglio europeo del gruppo di riflessione sul futuro dell'UE 2030, cit., p. 4.

15 Ibidem. 
Mario Draghi, in sede di Assemblea della Banca d'Italia, ${ }^{16}$ constatava come la crisi e la sua gestione avessero disatteso le aspettative degli estensori di quel rapporto. Pure in presenza di dati "oggettivi" favorevoli al confronto di quelli relativi a sistemi comparabili (deficit di bilancio previsto - per il 2011 - pari al 4,5\% del Pil europeo, valore inferiore di oltre la metà rispetto a quelli statunitense e giapponese; debito pubblico pari all' $88 \%$ del Pil, quindi anch'esso inferiore a quello statunitense e di molto a quello giapponese; bilancia dei pagamenti corrente prossima al pareggio, prevedibile consolidamento della ripresa produttiva), la prova che l'Unione economica e monetaria si trovava a fronteggiare era tuttavia la "...più difficile dalla sua creazione" ${ }^{17}$ In due successive affermazioni il Governatore chiarirà le ragioni di fondo della "fragilità" dell'Unione di fronte alla crisi, alle sue conseguenze e alle politiche di contrasto attuate e da attuare. La prima sottolinea l'insufficiente coordinamento delle politiche di bilancio, nei seguenti termini: "La sorveglianza europea sulle politiche di bilancio nazionali, indebolita a metà dello scorso decennio su iniziativa dei tre più grandi paesi... [Germania, Francia e Italia, n.d.r.]...si è dimostrata carente proprio nel momento in cui diventava essenziale" ${ }^{18}$ La seconda mette in relazione $\mathrm{i}$ successi raggiunti in campo monetario con gli effetti distorsivi che di quegli stessi successi erano la conseguenza, nei seguenti termini: "Per lungo tempo la moneta unica ha velato le differenze tra paesi membri nelle condizioni di fondo e nelle politiche economiche, l'assenza di regole comuni realmente vincolanti. Per lungo tempo $i$ premi per il rischio non hanno rivelato la verità ... [corsivo non nel testo, n.d.r.]". ${ }^{19}$

Quanto agli interventi di sostegno ai paesi in difficoltà, il 2010 aveva visto, nel marzo, la predisposizione di un meccanismo di supporto a favore degli Stati membri dell'area euro basato su prestiti bilaterali. Nel maggio venivano definiti due ulteriori strumenti temporanei di intervento/assistenza, lo European Financial Stabilisation Mechanism (EFSM) e, successivamente, lo European Financial Stability Facility (EFSF). Sarà poi nel marzo 2011 che il Consiglio europeo definirà le caratteristiche dello

16 Banca d'Italia, Considerazioni finali, Assemblea Ordinaria dei Partecipanti, Roma, 31 maggio 2011.

17 Ivi, p. 7.

18 Ibidem.

19 Ibidem. 
European Stability Mechanism (ESM), una vera e propria organizzazione internazionale destinata a sostituire l'EFSF a partire dal luglio 2013 e dotata di una capacità di prestito pari a 500 miliardi.

La predisposizione di meccanismi di sostegno finanziario progressivamente più cospicui sotto il profilo del potenziale di intervento, oltreché strutturalmente più "multilaterali", accompagna il succedersi di situazioni di tensione. Nel maggio del 2010 la Grecia otterrà il sostegno (vincolato) sia del Fondo Monetario Internazionale sia dei paesi dell'area euro. A fine novembre sarà la volta dell'Irlanda. Nell'aprile 2011 toccherà al Portogallo. A riprova della stretta connessione che viene a instaurarsi fra stato dei conti pubblici di un paese membro, robustezza del suo sistema bancario, grado di incisività dei poteri comunitari e necessità del loro rafforzamento, nella Relazione della Banca d'Italia per il 2010 si legge, a commento degli avvenimenti dell'anno precedente, che "Il sistema di sorveglianza multilaterale non disponeva di strumenti incisivi per la prevenzione e la correzione di squilibri di tipo macroeconomico. Mancavano inoltre meccanismi di intervento per le situazioni di grave crisi finanziaria di uno Stato membro...". ${ }^{20}$

4. Crisi, modalità di contrasto, strutture decisionali "a tendere", modalità e tempi per attuarle o quantomeno per approssimarvisi, erano dunque, alla data dell'incontro pavese, componenti stabili del contesto politico-economico e del dibattito che andava sviluppandosi: l'aggravarsi delle tensioni accentuava inoltre il convincimento che non soltanto fosse urgente intervenire, ma che modalità e meccanismi di intervento dovessero essere quanto più possibile prossimi proprio agli assetti "a tendere" individuati: il fatto poi che su questa seconda necessità - e su quegli assetti - non si desse, come tuttora non si dà, unanimità di propositi era, come tuttora è, fattore non secondario di tensioni ulteriori. Come già il suo predecessore, l'attuale Governatore della Banca d'Italia Ignazio Visco, rivolgendosi per la prima volta, nel maggio 2012, all'Assemblea della Banca d'Italia ${ }^{21}$ ribadiva nei seguenti termini un concetto già espresso da Draghi: "Inerzia politica,

20 Banca d'Italia, Relazione Annuale, Assemblea Ordinaria dei Partecipanti, Roma, 31 maggio 2011, pp. 63-4.

21 Banca d'Italia, Considerazioni finali, Assemblea Ordinaria dei Partecipanti, Roma, 31 maggio 2012. 
inosservanza delle regole e scelte economiche errate hanno favorito l'emergere di squilibri interni, a lungo offuscati dall'euro e ignorati dai mercati... [corsivo non nel testo, n.d.r.]..., che rischiano oggi di mettere a repentaglio l'intera costruzione". ${ }^{22} \mathrm{E}$ ricordava i lineamenti dell'assettoobiettivo dell'Unione nei seguenti, significativi termini: "Se si guardasse all'area dell'euro come a un'entità unitaria, nella forma ad esempio di uno Stato federale, non emergerebbero allarmi sulla tenuta del suo impianto monetario e finanziario, pur nella preoccupazione per le ripercussioni della crisi su ciclo economico, intermediari e mercati. Ma una unione politica in Europa ancora non c'è. Questo rende alla lunga l'unione monetaria più difficile da sostenere; sono necessari passi avanti concreti nella costruzione europea; va definito un percorso che abbia nell'unione politica il suo traguardo finale, scandendone le singole tappe...". ${ }^{23} \mathrm{D}$ 'altra parte: "L'economia dell'area dell'euro è da tempo integrata; comprende oltre 300 milioni di cittadini, quasi venti milioni di imprese. Considerata nel suo insieme, ha conti con l'estero bilanciati e un debito del settore pubblico previsti quest'anno...[il 2012, n.d.r.]...poco sopra, rispettivamente, il 3 e il $90 \%$ del PIL; famiglie con una ricchezza finanziaria lorda che è 3 volte il loro reddito disponibile annuo e un indebitamento pari al reddito; un debito finanziario aggregato delle imprese pari al prodotto di un anno. Sono dati che configurano un'economia solida ed equilibrata, per molti aspetti più di altre aree avanzate del mondo" ${ }^{24}$ È proprio in ragione di tali elementi che "Si avverte la mancanza di fondamentali caratteristiche di una federazione di Stati: processi decisionali che favoriscano l'adozione di politiche lungimiranti, nell'interesse generale; risorse pubbliche comuni per la stabilità finanziaria e per la crescita; regole davvero condivise e azioni concordate e tempestive sul sistema finanziario e sulle banche. Sono compiti e condizioni che esorbitano dalla sfera d'azione del Sistema europeo di banche centrali: investono responsabilità politiche, nazionali e comunitarie". ${ }^{25}$ Ancora una volta, poi, alle considerazioni formulate dal Governatore corrispondevano, nel corpo della Relazione dell'Istituto, sia un apprezzamento complessivo degli sforzi compiuti nella direzione di assetti istituzionali più adeguati alle necessità, sia la con-

\footnotetext{
22 Ivi, p. 15.

23 Ivi, p. 14.

24 Ibidem.

25 Ivi, p. 15.
} 
statazione di dati "oggettivi" di massima favorevoli. Ma, una volta ancora, la mancanza di progressi irrevocabili - e percepiti come tali - nella direzione sia di "processi decisionali che favoriscano l'adozione di politiche lungimiranti, nell'interesse generale", sia di "regole davvero condivise e azioni concordate e tempestive sul sistema finanziario e sulle banche", si è resa evidente, determinando la necessità di ulteriore sostegno finanziario a favore di Grecia, Irlanda e Portogallo.

5. Collocati in posizione cronologicamente mediana fra la conclusione dei lavori del Gruppo di riflessione sul futuro dell'UE e la Relazione Annuale della Banca d'Italia del 2012 (la prima di Ignazio Visco) e pressoché contemporanei a quella dell'anno precedente (l'ultima di Mario Draghi), i contributi raccolti nel volume partecipano di tesi, convincimenti, previsioni, preoccupazioni, auspici, tecnicismi che si rinvengono, variamente distribuiti, anche nei documenti richiamati, sia in quelli che hanno preceduto l'incontro di Pavia sia in quelli successivi: l'ispirazione di fondo, marcatamente avversa all'approccio intergovernativo tuttora prevalente (e favorevole invece, per dirla con De Grauwe, a procedure di decisione idonee a internalizzare le esternalità, che sarebbe poi il compito di ogni Governo), sembra inoltre avere tratto forza di convinzione dall' ambiente intellettuale, quello pavese, nel quale l'incontro si era svolto, e che è stato sinteticamente rievocato in apertura.

A due dei contributi contenuti nel volume, rispettivamente di Alberto Botta e di Marcello Messori, dedicherò, in conclusione, un'attenzione particolare, precisando tuttavia che anche gli altri (di Alberto Majocchi, di Dario Velo, di Vincenzo Visco e di Jacques Ziller, nonché la Presentazione di Franco Osculati e la nota introduttiva di chi scrive) condividono la medesima impostazione di fondo. Alberto Botta, in particolare, propone un modello analitico utile a contrastare l' "ortodossia della virtù" attualmente prevalente in Europa, inquadrando in una prospettiva post-keynesiana (à la Minsky) la relazione fra crisi dei debiti sovrani e instabilità finanziaria. Le misure di salvataggio attuate a favore di istituzioni finanziarie in difficoltà danno luogo a comportamenti di mercato riconducibili alla categoria delle self-fulfilling expectations, innescando in successione "fuga" dai titoli pubblici, aumento dei rendimenti, aggravamento della gestione del debito, "avvitamento" recessivo del sistema; nel tentativo di rassicurare i mercati si pongono in essere politiche di bilancio restrittive che determinano un appesanti- 
mento degli stessi conti pubblici a danno delle prospettive di crescita del sistema ("prima priorità" secondo il Gruppo di riflessione sul futuro dell'UE); il perseguimento dell'obiettivo della crescita richiederebbe, all'opposto, politiche espansive finalizzate al miglioramento di produttività e competitività, ma queste appaiono inattuabili proprio in ragione delle conseguenze che - prevedibilmente - esse determinerebbero sui comportamenti degli operatori. Gli eurobond, in quanto strumento di integrazione fiscale fra i membri dell'Unione, indispensabile all'attuazione di politiche di bilancio idonee a contrastare le conseguenze reali delle crisi finanziarie promuovendo la crescita di lungo periodo, determinerebbero - e simboleggerebbero - proprio quella discontinuità istituzionale che (in quanto manifestazione della "volontà irrevocabile di preservare la moneta unica” auspicata dal Governatore Visco) è condizione indispensabile per superare l'asimmetria strutturale caratteristica dell'assetto attuale dell'Unione: un'esperienza "costituzionale" davvero singolare, quella europea, in quanto caratterizzata dalla Costituzione come obiettivo e non invece, come nell'esperienza statunitense, dalla Costituzione come presupposto.

Al tema degli eurobond, strumento finalizzato alla provvista di risorse finanziarie necessarie alla realizzazione di un piano di rilancio della produttività e della competitività europee e alla produzione di quei "beni pubblici europei" che sono condizione di crescita dell'Unione, è poi specificamente dedicato il saggio di Marcello Messori. Questo ripercorre le vicende di crisi di alcuni debiti sovrani europei e della conseguente attivazione di meccanismi europei di stabilizzazione finanziaria, sottolineandone la non adeguatezza a risolvere il problema del "debito sovrano europeo" in contesti di perduranti tensioni di mercato e di difficoltà crescenti in settori rilevanti di alcuni sistemi bancari. Viene proposta, come soluzione strutturale, l'istituzione di una European Debt Agency, EDA, operante secondo regole (di particolare interesse appare il proposto meccanismo dell'asta inversa) per quanto possibile "di mercato", idonee a massimizzare la compatibilità fra gli interessi - tuttora divergenti - dei paesi "periferici" e di quelli "centrali", in altri termini del Sud e del Nord dell'Europa.

6. Il periodo intercorso fra la Relazione Annuale della Banca d'Italia del 2012 e la redazione della presente nota ha visto succedersi, alternarsi e confliggere - enfatizzati dalla percezione di una crescente 
generalizzata precarietà del sistema internazionale delle interdipendenze - numerosi e rilevanti eventi, molti dei quali hanno assunto - in Europa - la forma di riunioni e consultazioni di "vertice". Ma gli elementi costitutivi, anzi costituzionali, dell'assetto "a tendere" dell'Europa stanno già tutti nelle Considerazioni finali del Governatore Visco del 2012 richiamate sopra: e tutti sono sintetizzabili nell'unione politica del continente come obiettivo del quale prevedere e realizzare le tappe e nella quale - per definizione - ricomprenderne i molteplici elementi, cioè processi decisionali compatibili con il perseguimento dell'interesse generale continentale, messa in comune di risorse in vista di tale obiettivo, regolamentazione "europea" dei sistemi bancari e finanziari. La discussione non può quindi che rivolgersi con particolare impegno proprio agli eurobond in quanto, operativamente e simbolicamente, atto forte e strutturale di "messa in comune" e, in quanto tali e al di là dei tecnicismi, "segno di contraddizione" fra visioni divergenti della costruzione europea. È proprio per questa ragione che, a oltre un anno dall'incontro di Pavia, riproporre (unitamente ad altri) i contributi discussi in quella sede significa ribadire un elemento centrale dell'unificazione europea, ma soprattutto andare al "cuore" del problema condividendo l'opinione secondo cui "Chi è in prima linea per salvare l'euro e fronteggiare la crisi sta...prendendo coscienza della natura sostanzialmente politica delle difficoltà che l'Europa deve fronteggiare e del fatto che il pesantissimo e prolungato attacco speculativo contro l'euro dei mesi scorsi è da imputare innanzitutto alla fragilità di un'unione monetaria che, priva di strumenti di governo efficaci, non riesce ad affermare le potenzialità dell'area che rappresenta e rischia di farsi trascinare nel baratro dalle situazioni di maggiore debolezza" ${ }^{26}$

26 Publius, Lettera Europea, maggio 2012. Significativa la seguente presa di posizione di Stiglitz a commento di un recente vertice europeo: "Come un detenuto nel braccio della morte, l'euro ha ottenuto, all'ultimo minuto, l'ennesimo rinvio dell'esecuzione. Sopravviverà. I mercati hanno festeggiato, come hanno fatto dopo ognuno dei precedenti vertici 'anticrisi', salvo poi accorgersi, immancabilmente, che i problemi di fondo non erano stati affrontati. I leader europei hanno finalmente capito che il metodo fai da te, con l'Europa che presta denaro alle banche per salvare gli Stati e agli Stati per salvare le banche, non funziona... Inquieta profondamente che i leader europei abbiano impiegato tutto questo tempo per rendersi conto di qualcosa di tanto ovvio" (J.E. Stiglitz, L'euro e la pena sospesa, "la Repubblica", 13 luglio 2012, tradotto da Id., The Euro's Latest Reprieve, www.project-syndicate.org). 
L'alternativa conduce a inseguire ogni emergenza con interventi sempre meno incisivi pur se caratterizzati da "architetture" sempre più sofisticate: qualora percorressimo questa strada, quando cioè seguissimo una "politica di timidezza e di prudenza... del rallentatore...infine di sfiducia", saremmo sempre "...'en arrière d'une année, d'une idée et d'une armée' come l'esercito degli Asburgo". ${ }^{27}$ D'altra parte la via maestra è indicata, e proprio con riferimento al problema dei debiti sovrani, dalle vicende della federazione fra tutte paradigmatica, gli Stati Uniti d'America e in particolare dagli esiti dell'acceso dibattito svoltosi fra Alexander Hamilton e Thomas Jefferson nel corso degli anni '90 del Settecento. Furono le tesi 'sviluppiste' e 'industrialiste' di Hamilton (esposte nei Reports del 1790-91 sul credito pubblico, sulla Banca centrale e sulle manifatture) a prevalere alla fine su quelle, conservatrici e di matrice mercantilista da "Repubblica di liberi agricoltori", di Jefferson. Da quella vicenda emersero - nel 1791 - sia la First Bank di Filadelfia sia, più in generale, un assetto forte di Stato federale. Fra le tesi di Hamilton, che alla fine prevalsero, fondamentale si rivelò proprio l'assunzione dei debiti degli Stati da parte dell'Unione, alla quale si sarebbero affiancati tanto una Banca nazionale autorizzata a emettere moneta e a fare credito al governo federale quanto un governo federale che assegnava a sé stesso l' obiettivo di favorire lo sviluppo delle imprese manifatturiere in vista dell'indipendenza economica e del progresso generale dell'Unione. ${ }^{28}$

27 Le due citazioni riportate in conclusione sono tratte dall'intervento del Consultore socialista Piero Della Giusta durante il dibattito sulla relazione del Ministro del Tesoro, il liberale Epicarmo Corbino, in sede di Consulta Nazionale nel 1946; cfr. Consulta Nazionale, Assemblea plenaria (seduta di martedì 22 gennaio 1946), Tipografia della Camera dei Deputati, p. 482. Corbino aveva rivendicato quale principio ispiratore della propria azione di governo la 'politica della lesina' praticata dal suo lontano predecessore Sidney Sonnino, un antesignano del "rigore" di oggi.

28 Per una recente, accurata ricostruzione di quel dibattito cfr. G. Bottaro, Alexander Hamilton: potere politico e potere economico in America in "Il Politico", 2012, n, 2, pp. 15- 32. Vi si conclude, fra l'altro (p. 32), che "Il vertiginoso sviluppo industriale e finanziario dei successivi due secoli non si sarebbe realisticamente potuto estrinsecare senza il forte impulso determinato, nell'ultimo scorcio del Settecento, dalla ferrea volontà di Hamilton di trasformare le deboli repubbliche agrarie americane in un forte e moderno Stato federale". 